\title{
A New Design of UHF RFID Tag Based on Split Ring Resonator For Moroccan Frequency Band
}

\author{
Latifa EL AHMAR ${ }^{1}$, Ahmed ERRKIK ${ }^{1}$, Jamal ZBITOU ${ }^{1}$,Ilham BOUZIDA ${ }^{2}$ and Brahim \\ LAKSSIR $^{2}$.
}

elahmarlatifa@gmail.com, ahmed.errkik@uhp.ac.ma, zbitouj@gmail.com, i.bouzida@mascir.com and b.lakssir@mascir.com

1 MEET laboratory, FST of Settat UH1 and 2 Packaging laboratory,MAScIR.

\begin{abstract}
In this paper, a new proposed design is presented for UHF RFID antenna matched to the Moroccan frequency band authorized by the ANRT. A proposed structure is inspired from circular split ring resonator theory, the geometry of the RFID antenna is double sided. Front side presenting a modified SRR while the back side is a circular ground plane. In order to achieve a good matching input impedance between the antenna and the impedance of the UHF chip, the structure is combined a modified SSR with stubs. The EM simulation of the proposed antenna done by EM Solver on an FR-4 substrate. The use of SRR permit to obtain good results of simulation with a reflection coefficient about $26,146 \mathrm{~dB}$ at the Moroccan frequency band around $868 \mathrm{MHz}$, a good read range and reduced size of RFID antenna were obtained with physical dimensions of $32 \times 18 \times 0.87 \mathrm{~mm}^{3}$ which presents $62.9 \%$ in comparaison with diemnsions of $\lambda / 4$ length.
\end{abstract}

Keywords: SRR, UHF, RFID, tag.

\section{Introduction}

Radio-Frequency identification is a technique which allows the exchange of the data between the RFID tag and the base station. The introduction of this technology motivated future researches to integrate RFID into various applications by using labels or transponders. These tags are the heart of RFID system that includes a base station to read the transponders. RFID system is based on two main elements, as shown in Figure 1.

RFID tags: composed of antenna connected to a chip activated by electromagnetic wave coming from reader antennas.

RFID base station: includes two parts, RFID reader as a principal device for communication with RFID tag via antennas and a Processing unit (PU) connected to the reader. The PU represents a software to initiate read/write operation, it can be a stationary or mobile platform to save results. [1]

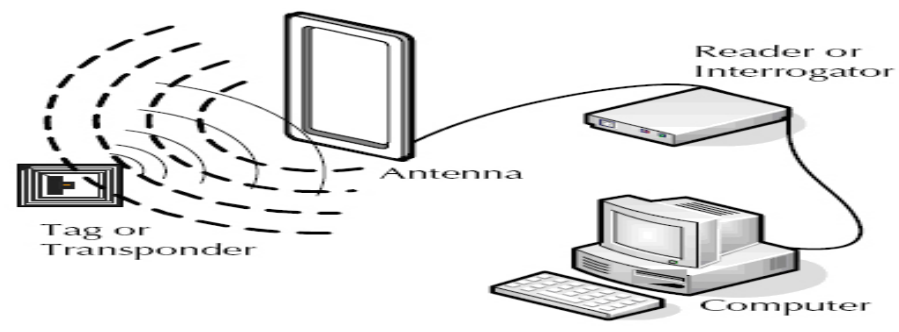

Fig. 1 Basic RFID system 
The revolution of RFID is manifested by growing demand in industry. This technology represents fundamental proprieties as low cost and efficiency of RFID devices, also, it's able to add more functionalities by integrating sensors to tags and get more information (humidity, temperature...etc). In the market, there are three types of transponder: Passive tags that use electromagnetic waves to feed the chip, active tags are powered by external battery and the last technology of tags called BAP which combined active and passive tags. For RFID technology, specified frequency bands are standardized, there are Low-Frequency band (LF) (125-134 $\mathrm{KHz})$, the High-Frequency band (HF) $(13.56 \mathrm{MHz})$, the Ultra High Frequency (UHF) (860-960 $\mathrm{MHz})$ and microwave frequency band $(2.4 \mathrm{GHz}$ and $5.8 \mathrm{GHz})$.

This work presents new structure of RFID tag adapted to Moroccan UHF band based on a modified circular Split Ring Resonator (SRR). The SRR show considerable interest due to their unique properties and also their physical dimensions that are less than $\leq \lambda / 10$. The flow of this paper is : the second section demonstrates the theory of the SRR structure and the design of modified SRR, the 3rd section shows results of the EM simulation of the new structure and in last section is a conclusion of work.

\section{Theoretical and Design}

This section is an introduction of split ring resonator theory, it describes the final geometry of the RFID tag based on circular modified SRR at Moroccan frequency $(868 \mathrm{MHz})$.

\subsection{Electrical equation of circular SRR:}

The circular Split ring resonator is commonly used for antenna design and passive circuits, thanks to their proprieties and also the simple design. The circular SRR behaviors is determined by the electrical elements of the equivalent circuit $\mathrm{L}$ and $\mathrm{C}$ which gives the resonance frequency according to the physical parameters of the structure, illustrated on Figure 2.
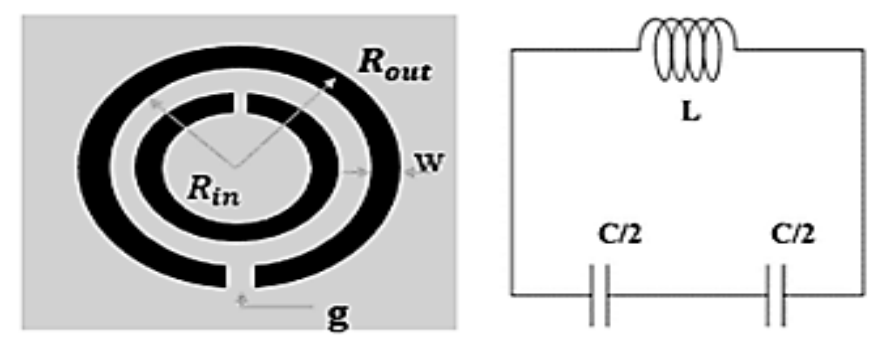

Fig.2 Circular Split Ring Resonator.

The resonance frequency is determined by (1):

$$
f_{r}=\frac{1}{2 \pi \sqrt{\mathrm{LC}}}
$$


L inductance of the electrical circuit is computed by (2), as referred in [5]

$$
\mathrm{L}=\mu_{0} \mathrm{R}_{\mathrm{m}}\left(\ln \left(\frac{8 \mathrm{R}_{\mathrm{m}}}{\mathrm{h}+\mathrm{w}}\right)-0.5\right. \text {. }
$$

With $\quad \mu_{0}$ : Permeability of free space, $1.256 \times 10^{-6}\left[\mathrm{~m} . \mathrm{Kg} \cdot \mathrm{s}^{-2} \cdot \mathrm{A}^{-2}\right]$.

$R_{m}$ : Average radius $\mathrm{R}_{\mathrm{m}}=\mathrm{R}+\mathrm{w} / 2$.

Total capacitance of the SRR structure is composed of two types of capacitance as mentioned in [5] by equation (3):

$$
\mathrm{C}=\mathrm{C}_{\text {gap }}+\mathrm{C}_{\text {surf }}
$$

With

Cgap is a capacitance due to the effect of the gap on structure computed by (4):

$$
C_{\text {gap }}=\varepsilon_{0}\left[\frac{w h}{g}+\frac{2 \pi h}{\ln \left(\frac{2.4 h}{w}\right)}\right] \text {. }
$$

With

$\mathrm{w}$ is a metal width, $\mathrm{h}$ is the height of the susbtarte, $\mathrm{g}$ is width of the gap and, $\boldsymbol{\varepsilon}_{\mathbf{0}}$ Permittivity of free space $\varepsilon_{0}=8.85418781710^{-12} \mathrm{~F} . \mathrm{m}^{-1}$.

And surface capacitance $\boldsymbol{C}_{\text {surf }}$ is contributed by the presence of the electric charges on metallic surface of the resonator. As denoted in equation (5):

$$
\mathrm{C}_{\text {surf }}=\int_{\theta_{\mathrm{g}}}^{\pi} \frac{\sigma \mathrm{Rd} \theta}{\mathrm{V}}=\varepsilon_{0} \mathrm{~h} \int_{\theta_{\mathrm{g}}}^{\pi} \frac{\cot \left(\frac{\theta}{2}\right)}{\pi-\theta} \mathrm{d} \theta \simeq \frac{2 \varepsilon_{0} \mathrm{~h}}{\pi} \ln \left(\frac{4 \mathrm{R}}{\mathrm{g}}\right) .
$$

With

$$
\theta_{\mathrm{g}}=\arcsin \left(\frac{\mathrm{g}}{2 \mathrm{R}}\right)
$$

$\sigma \quad$ Surface charge density, $\sigma=\frac{\varepsilon_{0} V_{0}}{2 \pi R} \cot \left(\frac{\theta}{2}\right)$ and V voltage on the gap, it's given by $V=\frac{c}{\pi}(\pi-\theta)$.

\subsection{Anetnna Design :}

The tag is designed as so to resonate at $868 \mathrm{MHz}$ and to prove a good performance, the proposed antenna design starts with a simple circular split ring resonator and two stubs are added in order to realize a good matching impedance between the antenna and the UHF chip. The ground plane is used to make the antenna more directive. The geometry is a double-sided antenna designed on a FR-4 substrate with a dielectric permittivity of 4.4, loss tangent of 0.025 and a thickness of $0.8 \mathrm{~mm}$, as illustrated on Figure 3.
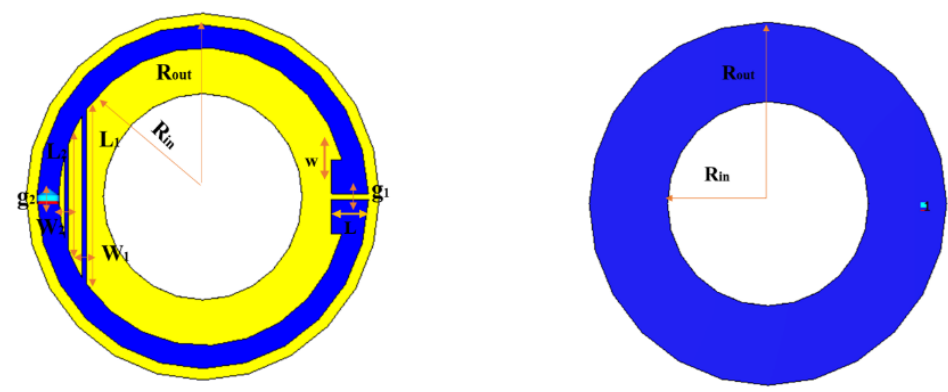

Fig.3 Proposed tag at $868 \mathrm{MHz}$. 
To achieve a maximum transmitted power from the antenna to the UHF chip Alien H3 with a complex impedance at $868 \mathrm{MHz} \quad \mathrm{Z}_{\text {chip }}=27-\mathrm{j} 110$.In litturature the antenna impedance should be equal to the conjugate of the impedance of UHF chip and to be strongly inductive to reduce the capacity effect of the chip.

Based on theoretical equations of circular split ring resonator, the physical parameters of the design determine the resonance frequency, for our design, several works are done to reach optimal parameters allowing good results at Moroccan UHF bands. This structure allows easy control of the resonance frequency and also more flexibility in term of the dimension of proposed antenna for RFID applications that require small antenna's dimensions. The optimal parameters of proposed structure, are presented in table 1.

Table 1: Optimal parameters of the antenna proposed.

\begin{tabular}{|l|l|l|}
\hline Parameters & Value (mm) & $R_{\text {in }}=13$ \\
\hline Ring & $R_{\text {out }}=15$ & \multicolumn{2}{|c|}{$g=g_{1}=g_{2}=0.5$} \\
\hline Gap & \multicolumn{2}{|c|}{} \\
\hline Microstrip Lines & $\mathrm{L}_{1}=19$ & $\mathrm{~W}_{1}=0.39$ \\
\cline { 2 - 3 } & $\mathrm{L}_{2}=15$ & $\mathrm{~W}_{2}=0.32$ \\
\hline Stub & $\mathrm{L}=0.32$ & $\mathrm{~W}=1.35$ \\
\hline Ground Plane & $R_{\text {out }}=16$ & $R_{\text {in }}=9$ \\
\hline
\end{tabular}

The geometric parameters of the tag and resonance frequency are dependant as shown in [5], also the work is done to study resonance response of the structure which proves capability to reveal resonance at $868 \mathrm{MHz}$. In theory the Moroccan frequency represents a wavelength about $345.6 \mathrm{~mm}$, which is $10.8 \mathrm{x}$ of the greater diameter of the structure.

By projecting the theoretical expressions presented on [5] using optimal parameters shown on table 1 to calculate electrical elements presented in table 2.

Table 2: Electrical elements of the proposed tag.

\begin{tabular}{|l|l|}
\hline Parameters & Value(mm) \\
\hline L & $60 \mathrm{nH}$ \\
\hline C & $56.03 \mathrm{pF}$ \\
\hline $\mathrm{C}_{\text {gap }}$ & $24.6 \mathrm{pF}$ \\
\hline $\mathrm{C}_{\text {gap_totale }}$ & $49.2 \mathrm{pF}$ \\
\hline $\mathrm{C}_{\text {surf }}$ & $6.833 \mathrm{pF}$ \\
\hline
\end{tabular}

Microstrip lines and stubs are characterized by electrical impedance presented in table $\mathbf{3}$. 
Table 3: Characteristic impedances of the proposed structure.

\begin{tabular}{|l|l|l|l|}
\cline { 2 - 4 } \multicolumn{1}{c|}{} & Microstrip Line 1 & Microstrip Line 2 & Stubs \\
\hline Characteristic impedance & $103.4 \Omega$. & $96.32 \Omega$. & $54,0421 \Omega$ \\
\hline
\end{tabular}

\section{Simulation and discussion:}

According to the results of the EM simulation using CST mws , this RFID tag present a good adaptation with a reflection coefficient of $-26,146 \mathrm{~dB}$ at the Moroccan frequency $(868 \mathrm{MHz})$ as follows in Figure 4 and a bandwidth of $1.94 \mathrm{MHz}$ as follows in Figure $\mathbf{5}$, compared to the literature a S11 must be less than $-10 \mathrm{~dB}$ at a desired resonance frequency so the proposed tag is accepted in term of incident power that is reflected back towards the source from the antenna, in Morocco the bands is very limited $0.5 \mathrm{MHz}$ while the structure give us a large band of $1.94 \mathrm{MHz}$.

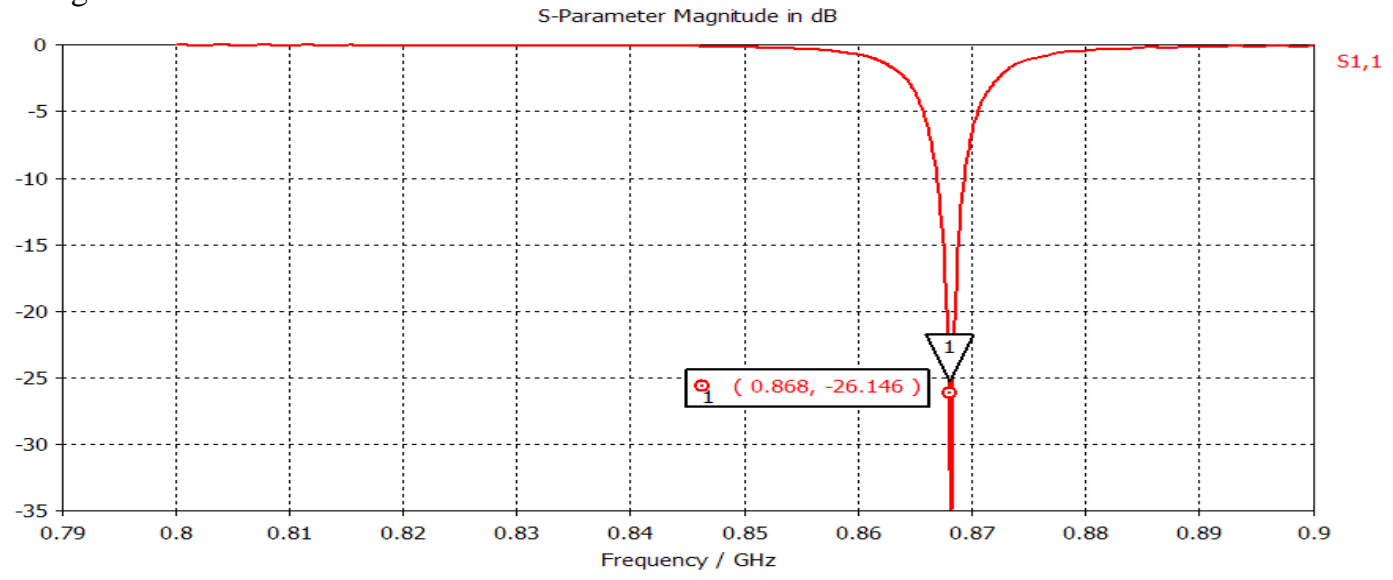

Fig.4 Simulated Reflection coefficient of the proposed RFID antenna.

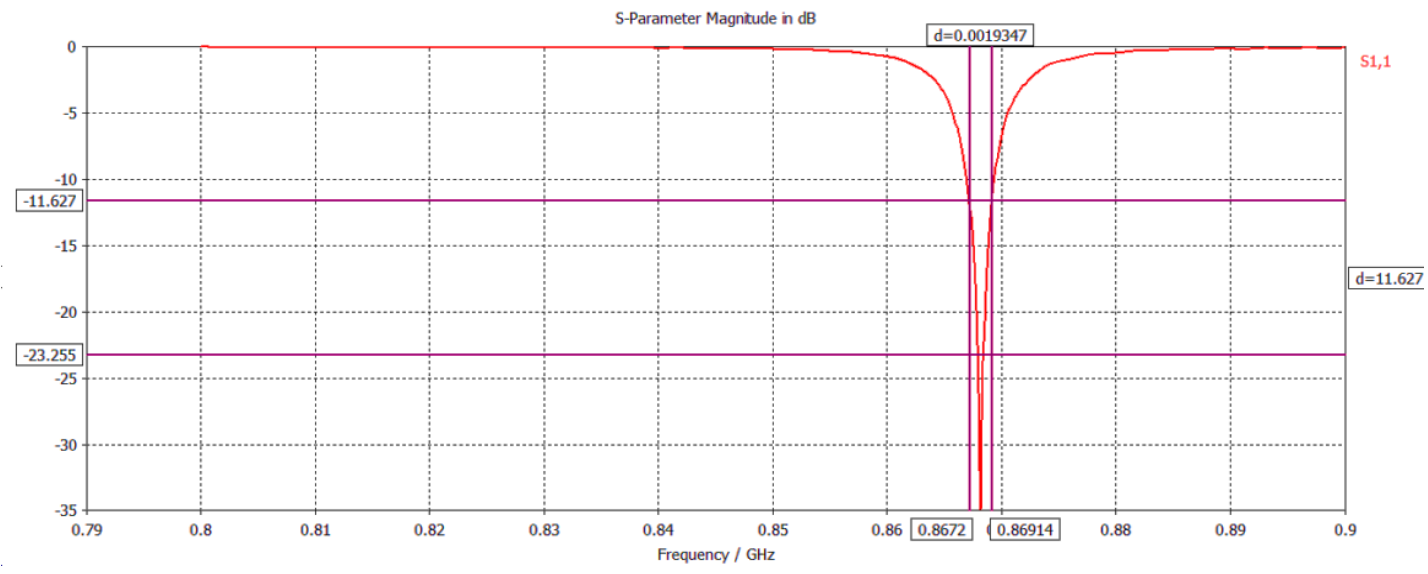

Fig.5 Bandwidth of the proposed RFID antenna. 
For the selection of an antenna we evaluate also the VSWR (Voltage Standing Wave Radio) , VSWR describes the power reflection from the antenna, the ideal case is when VSWR is equal to 1 which means no reflection power. In real cases the VSWR less than 2 is acceptable for a small antenna. In case of our RFID tag, VSWR is equal to 1.1 at $868 \mathrm{MHz}$ as shows in Figure 6, that is a good VSWR, in term of power that's represent $99.7 \%$ of the power transmission and $0.3 \%$ of the power reflection .

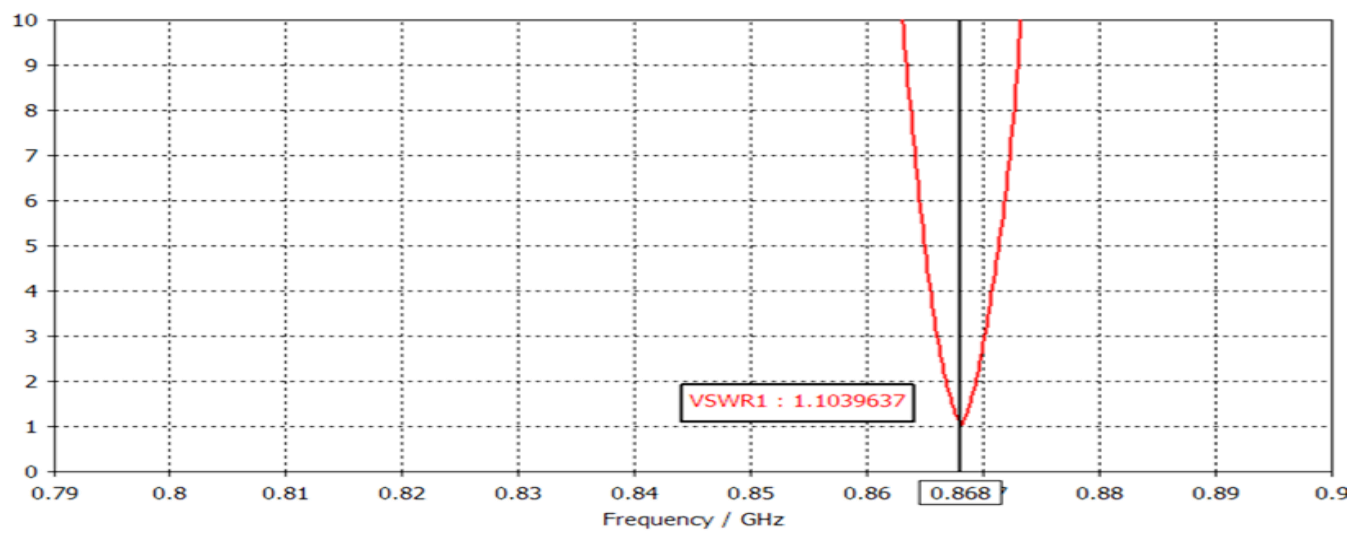

Fig.6 Simulated VSWR

The radiation pattern in the following Figure 7 shows the RFID tag having a maximum directivity equal to $1.545 \mathrm{dBi}$ at $868 \mathrm{MHz}$ and the distribution of the radiation is more concentrated in both gaps of the structure.

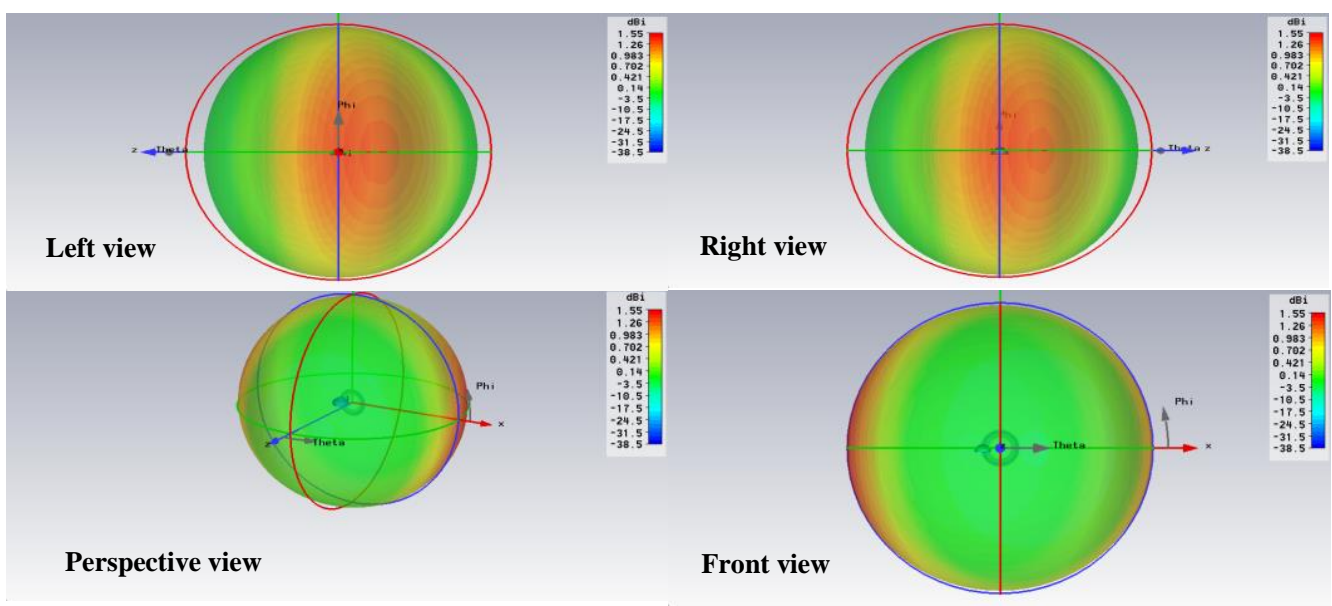

Fig.7 Radiation of the proposed RFID antenna

The read range is an important requirement of RFID tag design, it gives us an information about the maximum distance at which the tag receives the minimum threshold power required to turn on and scatter a signal back, and the maximum distance at which the reader can detect this return signal.Using the Friis free-space equation, we get to calculate the read range using 
MATLAB script.The proposed antenna evaluate a read range equal to $1,6 \mathrm{~m}$ which is the maximum distance that our proposed tag can communicate with the reader. As shown in Figure 8.

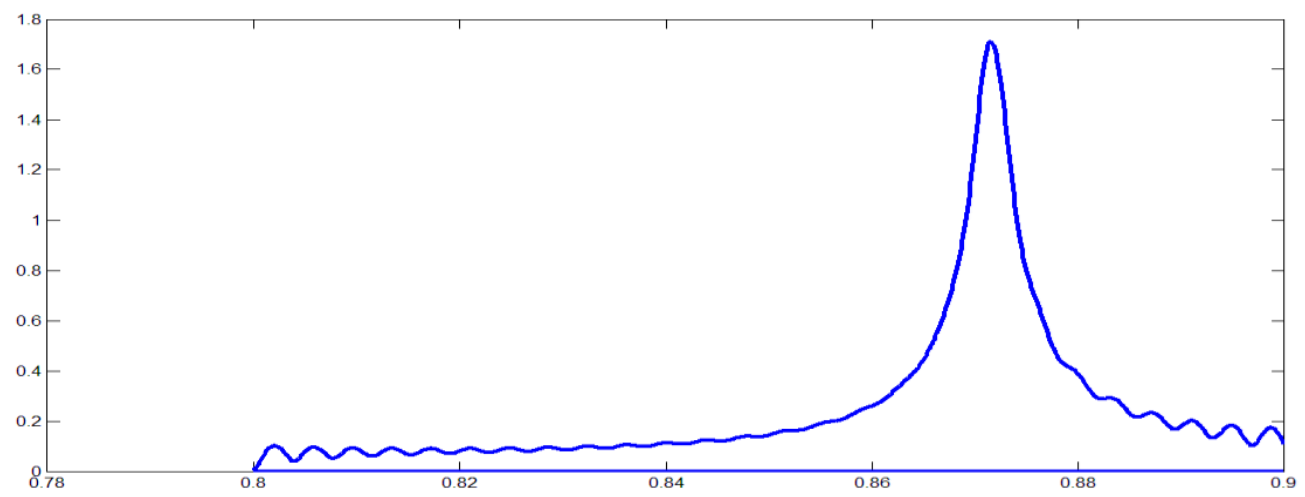

Fig.8 Read range of the proposed RFID antenna.

The proposed RFID tag having a negative gain at $868 \mathrm{MHz}$, as shown in Figure 9, this result is explained according to the literature to the size of the antenna that is too smaller than $\frac{1}{2}$ or $\frac{1}{4} \lambda$ ,in our case the size of the antenna is $32 \mathrm{~mm}$ less than $\frac{\lambda}{4}=86.4 \mathrm{~mm}$, we call it an electrically small antenna that is characterized by an antenna gain less than 1 .

Gain

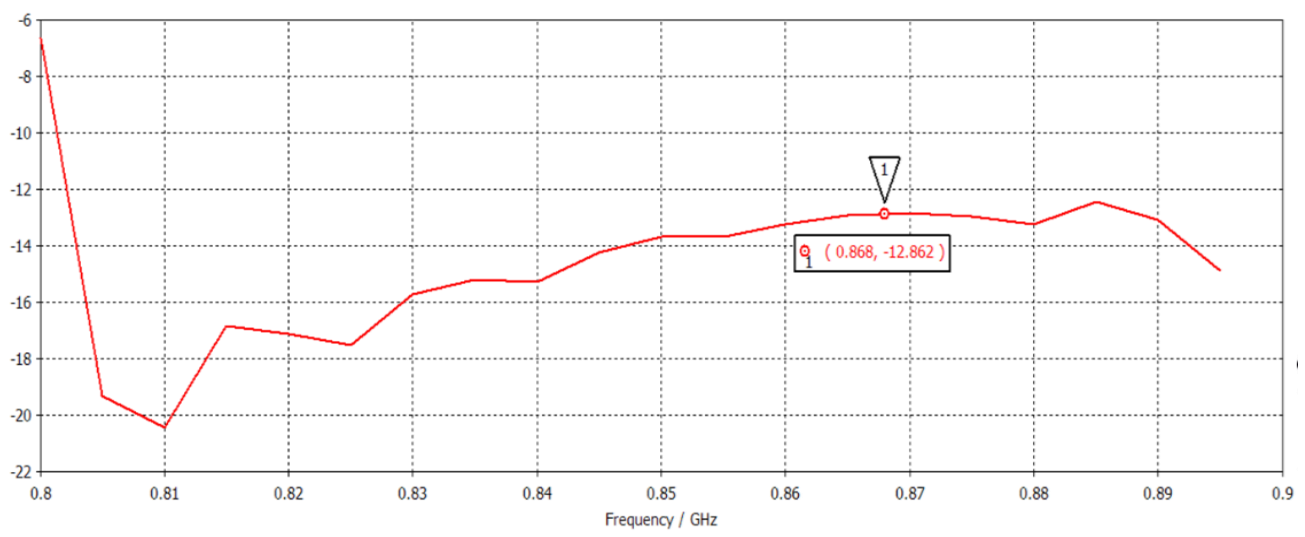

Fig.9 Gain Of the RFID tag at $868 \mathrm{MHz}$.

After the validation of the structure using CST mws and fixing the dimensions and knowing the performance of the RFID tag, the tag is fabricated as shown in Figure $\mathbf{1 0}$ manually in the lab. The FR-4 with $\varepsilon r=4.4, \tan \delta=0.025$ and thickness $=0.8 \mathrm{~mm}$ is used as the substrate for the tag. Copper is used as the conductor. 


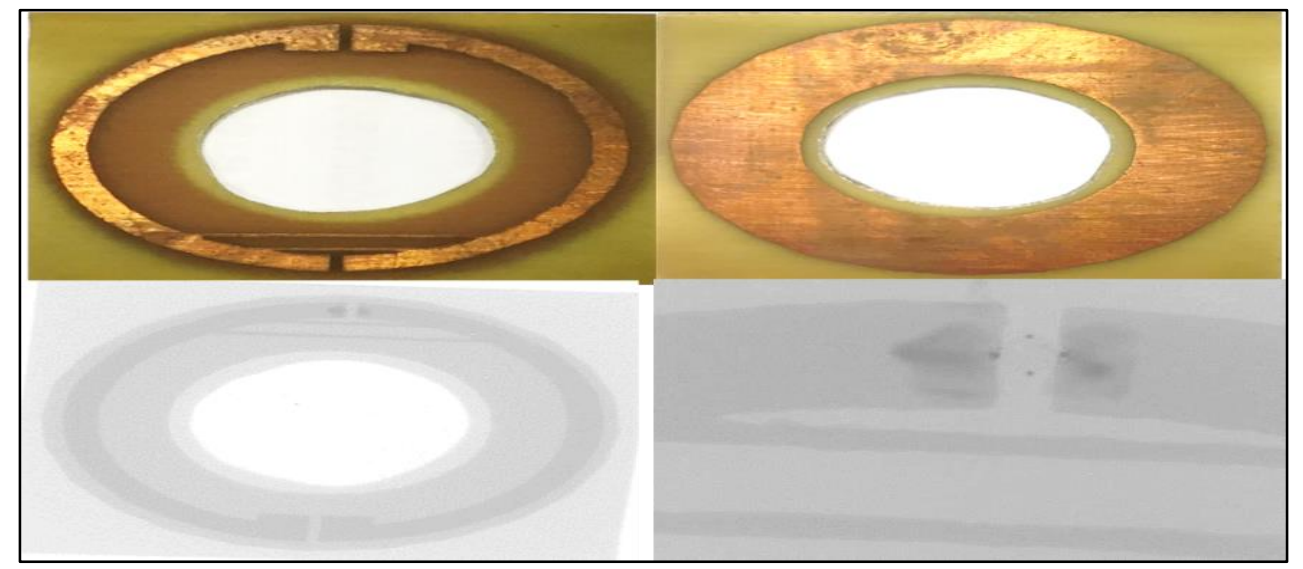

Fig.10 (i) Front view and bottom view of fabricated TAG antenna. (ii) Location of ALIEN H3 chip using X-Ray.

\section{Conclusion}

In this paper, a new contribution was given to the design of RFID tag based on metamaterials that are presented by a circular split ring resonator modified to achieve a good response at a Moroccan frequency and miniaturization of the design.Before fabricating the proposed tag, the EM simulation of the RFID antenna is demonstrated to achieve the requirements of design including matching input impedance, size and read range.

The RFID tag combined a simple split ring resonator and stubs to realize a good matching impedance. The result of the simulation presents a good matching of $-26,146 \mathrm{~dB}$ at $868 \mathrm{MHz}$ with a bandwidth of $1.94 \mathrm{MHz}$. The proposed RFID antenna has a small size $32 \times 18 \times$ $0.87 \mathrm{~mm}^{3}$ that comes to $62.9 \%$ of the theoretical size. The read range calculated using MATLAB agreed well with the test results by using a base station in the lab.

\section{References}

[1] B. Ghanem, "RFID Project Technical Report," 1970.

[2] A. E. Abdulhadi et al., "Antenna design for UHF RFID Tags: A review and a practical application," 2003.

[3] Advance Energy, "Impedance Matching," 2006.

[4] I. Antennas, P. Magazine, and V. Politecnico, "The art of UHF RFID antenna design : impedance matching and size-reduction techniques," 2008.

[5] Jabita and A.-N. Abiodun, "Design of Singly Split Single Ring Resonator for Measurement of Dielectric Constant of Materials using Resonant Method," Master's Thesis Electron. Telecommun. Univ. Gävle, no. June, 2013.

[6] N. H. Daud, A. Ismail, A. Reda, H. Alhawari, A. Sali, and M. F. A. Rasid, "Integration of Split Ring Resonators ( SRRs ) to UHF RFID Tag Antenna for Size Reduction," 2014.

[7] S. Zuffanelli, G. Zamora, P. Aguilà, F. Paredes, F. Martín, and J. Bonache, "Analysis of the Split Ring Resonator (SRR) Antenna Applied to Passive UHF-RFID Tag Design," IEEE Trans. Antennas Propag., vol. 64, no. 3, pp. 856-864, 2016.

[8] A. Ennajih, J. Zbitou, A. Errkik, and L. El Abdellaoui, "A New Design of UHF RFID Tag Antenna Based on Negative Index Metamaterial," pp. 1-6, 2017. 